\title{
Spike-in genomic DNA for validating performance of metagenomics workflows
}

\author{
Arvind Venkataraman*,1, Mirjana Parlov ${ }^{1}$, Ping Hu¹, Dan Schnell ${ }^{1,2}$, Xingtao Wei ${ }^{1}$ \& Jay P Tiesman ${ }^{1}$ \\ ${ }^{1}$ The Procter \& Gamble Company, 8700 Mason Montgomery Road, Mason, OH 45040, USA; ${ }^{2}$ Current affili- \\ ation: Cincinnati Children's Hospital, 333 Burnett Avenue, Cincinnati, OH 45229, USA
}

BioTechniques 65: 315-321 (December 2018) 10.2144/btn-2018-0089

Shotgun metagenomics is a powerful platform to characterize human microbiomes. However, to translate such survey data into consumer-relevant products or services, it is critical to have a robust metagenomics workflow. We present a tool - spike-in DNA - to assess performance of metagenomics workflows. The spike-in is DNA from two organisms - Alivibrio fischeri and Rhodopseudomonas palustris, in a ratio of 4:1 added to samples before DNA extraction. With a valid workflow, the output ratio of relative abundances of these organisms should be close to 4. This expectation was tested in samples of varying diversities $(n=110)$, and the mean ratio was $4.73(99 \% \mathrm{Cl}[4.0,5.24])$. We anticipate this tool to be a relevant community resource for assessing the quality of shotgun metagenomics workflows and thereby enable robust characterization of microbiomes.

Communities of microbes including bacteria, archaea, viruses and fungi play critical roles in sustaining and enabling life on earth by regulating global carbon cycles, powering agriculture by nitrogen fixation, providing metabolites that are integral to human health, and even remediating pollution [1-5]. Managing these microbial communities for a desired effect is an ongoing massive endeavor worldwide involving multiple universities and industries [6]. Critical towards this endeavor is generating an understanding of these complex microbial communities who is there, what functions/metabolism are they performing, and how are they carrying out these functions. Shotgun or untargeted metagenomics is a particularly powerful and revolutionary tool that can provide this fundamental information [7], because it involves direct sequencing and analysis of all the DNA extracted from a microbiome, be it soil, human, ocean or even bioreactors, thereby circumventing the requirement to isolate and cultivate each microbial species. Thus, metagenomics enables study of microbes in their own 'biomes'.
Despite the concept being simple and elegant, the workflow involved in performing metagenomics from a microbiome is not trivial [8]. The process is comprised of more than 40 steps involving DNA extraction, sample normalization, library preparation, library clean up, library normalization, sequencing and then bioinformatic analyses - with significant potential for introducing cumulative variability and bias. It is therefore critical to validate the entire workflow to ensure that the data derived using metagenomics is robust and can be used for discovery research and translation into products and services. This need has been echoed by multiple organizations such as the National Institute of Standards, and Technology-based International Microbiome and Metagenomics Standards Association, Genomic Standards Consortium, as well as recent peer-reviewed publications [9-11].

Accordingly, our goal was to develop a simple and inexpensive method to validate the entire metagenomics workflow. This spike-in approach for monitoring and validating our metagenomics workflow consists of adding DNA from known organisms in a defined ratio to the actual samples prior to cell lysis and then determining the ratio of relative abundances in which the spike-in is recovered at the very end of the workflow. We used DNA as the spike-in rather than whole cells because DNA quantitation is easier, more accurate and reproducible. Precise whole-cell count demands specialized equipment such as a flow cytometer [12], adds significant time and money to the endeavor, and is less reproducible. A whole-cell-based complex mock community of microbes was used to validate that the DNA extraction process lysed bacteria and fungi. DNA from Alivibrio fischeri and Rhodopseudomonas palustris was chosen as spike-in for a few different reasons. First, the GC contents of their DNA are widely different $-A$. fischeri has a GC content of $38 \%$ while $R$. palustris has a GC content of $65 \%$ - thereby allowing us to include potential GC-bias as a factor in validating our workflow. Second, the genomes of both these organisms are sequenced and publicly available ( $A$. fischeri ES114 - 4.2 Mb and R. palustris CGA0009 - $5.4 \mathrm{Mb}$ ); this facilitates bioinformatics analyses and normalization of

\section{METHOD SUMMARY}

A spike-in approach for monitoring and validating metagenomics workflows is presented. This consists of adding genomic DNA from known organisms in a defined ratio to the samples prior to cell lysis and then determining the ratio of relative abundances in which the spike-in is recovered at the very end of the workflow. 
sequencing reads to genome lengths. Finally, since the primary research focus for us is human-associated microbiomes, these organisms are typically not found in human microbiomes [13]. Our spike-in is also qualitatively different from the internal PhiX control because the PhiX control is used to monitor the quality of the actual sequencing (Q30 scores), and not the performance of the entire workflow.

While we focused on using this method for validating metagenomics workflows for human microbiome samples, the same principles should apply when extending this method to validate metagenomic workflows for characterizing microbiomes from any habitat. Needless to say, this will necessitate modification of input bacterial species as spikes. For instance, in the case of validating metagenomics workflows with marine microbiomes, possible choices could include well-characterized organisms from the Actinomyces and/or Clostridium genera, since these are not typically associated with marine habitats. This method is a community resource, because it is cheap, uses readily available supplies, and is easily implementable in a laboratory setting. Additionally, since metagenomic data analysis typically involves probing relative abundances, having a QC metric that in itself is a relative abundance measure is valuable.

\section{Materials \& methods}

Spike-in DNA

The spike-in consisted of genomic DNA from two marine bacteria $A$. fischeri strain ES 114 and $R$. palustris CGA0009 (ATCC 700601D-5 and BAA-98D-F respectively). The DNA was resuspended in $250 \mu$ of nuclease-free molecular-grade water and left at $4^{\circ} \mathrm{C}$ overnight. The DNA concentrations were quantified on a Qubit using the dsDNA High Sensitivity Kit. In addition, the size distribution of the DNA was assessed on an Agilent 4200 TapeStation. We wanted to ensure that the DNA from both organisms had a similar size distribution to prevent this from confounding our results further downstream. To create the spike-in mix, the DNA from $A$. fischeri and $R$. palustris was mixed in a 4:1 ratio using the Qubit-derived quantification. $5 \mu \mathrm{l}$ of the spike-in DNA mix contains $7.2 \mathrm{ng}$ DNA of $A$. fischeri and 1.8 ng DNA from $R$. palustris. Based upon these weights and genome sizes of the organisms (4.2 and
5.4 Mbp, respectively), the ratio of genome copy numbers is $~ 5.1$. However, since MetaPhIAn2 corrects for genome sizes, the ratio of relative abundances should be close to 4.

\section{Sample collection \& processing}

To test the performance of the metagenomics workflow with different kinds of samples, the spike-in mix described previously was added to: i) sterile phosphate-buffered saline; ii) defined mock communities of bacteria and fungi; and, iii) various mixtures of fecal material and the mock community microbial mixture. This enabled validation of the metagenomics workflow for samples of varying complexity ranging from spike-in alone to fecal material containing spike-in. There were at least eight replicates in each kind of sample.

The spike-in alone samples were prepared by adding $5 \mu$ of the spike-in mix described previously to $250 \mu$ of sterile phosphate-buffered saline. For the spike-in with the mock community, $5 \mu$ l of the spike-in mix was added to a $250 \mu$ l suspension of the mock community. This synthetic community contained a mixture of the following bacteria and fungi: Acinetobacter ursingii, Staphylococcus epidermidis, Enhydrobacter aerosaccus, Staphylococcus capitis, Staphylococcus hominis, Propionibacterium acnes, Micrococcus luteus, Corynebacterium tuberculostearicum, Corynebacterium jeikeium, Staphylococcus aureus, Malassezia globosa, Malassezia restricta, Malassezia furfur, Saccharomyces cerevisae, Candida albicans and Candida tropicalis. Each of these organisms was grown individually (tryptic soy broth and brain heart infusion for bacteria, Sabouraud dextrose broth for S. cerevisiae, C. albicans, C. tropicalis and mDixon for the Malassezia spp.). Using a $\mathrm{CFU} / \mathrm{ml}$ estimation, the liquid cultures of each organism were diluted down to $\sim 10^{6}$ $\mathrm{CFU} / \mathrm{ml}$ and equal volumes were mixed to generate the mock community. Fecal material was collected using the Covidien Commode Specimen Collector and a suspension of fecal material was prepared by adding $0.5 \mathrm{~g}$ of fecal material to $10 \mathrm{ml}$ of phosphate buffered saline and homogenizing this solution. This master fecal suspension and the mock community suspension were mixed in varying proportions on a volume to volume basis to create a series of samples ranging from 1/5th fecal + 4/5th mock community ( $1 \mathrm{ml}$ fecal suspension $+4 \mathrm{ml}$ mock community solution) to $4 / 5$ th fecal $+1 / 5$ th mock community (4 ml fecal suspension $+1 \mathrm{ml}$ mock community solution). The goal was to gradually increase the complexity of the background and thus 'stress-test' the metagenomics workflow using the spike-in. $5 \mu$ of the spike-in mix was added to $250 \mu$ l of these mixtures. We also added the spike-in to the master fecal suspension as well. The samples were prepared and DNA extraction was performed on the same day.

\section{DNA extraction}

DNA was extracted from the samples described in Section II using a modified version of the Agencourt DNAdvance DNA Isolation Protocol Kit (Beckman Coulter A48706). The extractions were performed manually in a 96-well plate. The sample locations in the 96-well plate were randomized to ensure that differences in spike-in performance between sample types was not systematic. The DNA extraction method is described in detail in the supplementary information. Briefly, it consisted of bead beating, protein digestion and DNA purification with magnetic beads. This DNA was quantified using a Qubit dsDNA high-sensitivity kit and then stored at $-20^{\circ} \mathrm{C}$ before further processing.

\section{Library preparation \& PCR clean-up}

The location of the resulting DNA from Section III was randomized again on a new Hard Shell 96-well PCR-plate. The DNA was normalized to $0.2 \mathrm{ng} / \mu \mathrm{l}$ and processed through the Nextera-XT library preparation kit as per the manufacturer's instructions. Briefly, tagmentation was performed and 12 cycles of PCR were performed to add the i7 and i5 (index), sequencing and common adapters (P5 and $\mathrm{P} 7$ ) required for cluster generation and sequencing. At this point the DNA was stored at $4^{\circ} \mathrm{C}$ overnight. On the following day, the library DNA was purified and size selection conducted with 0.6x Agencourt AMPure XP beads. The resulting DNA was quantified on a Qubit using the dsDNA High Sensitivty kit and also run on an Agilent Bioanalyzer to confirm that the size of the DNA ranged from 350-800 bp. The molarity was calculated using the average base size output of the Agilent Bioanalyzer and the Qubitderived concentration. 
Library denaturing, normalization \& sequencing

The libraries from Section IV were normalized to $2 \mathrm{nM}$ and equal volumes pooled together. This was also run on the Agilent BioAnalyzer and concentration quantified via Qubit in order to confirm that the molarity of the pool was also $2 \mathrm{nM}$. We used the standard normalization method as outlined in Illumina's protocol and PhiX DNA was added at 1\% to serve as an internal sequencing control. These libraries were then sequenced on two high-output flow cells $(2 \times 150$ paired end reads) on a NextSeq 500 instrument. The cluster density was $180 \mathrm{~K} / \mathrm{mm}^{2}, 85 \%$ of the reads had a $Q$-score $\geq 30$, and the data by cycle intensity did not drop below 1000 (except between reads 1 and 2, to be expected).

\section{Bioinformatic analyses}

Sequence bases with quality score below 30 were discarded from both $3^{\prime}$ and $5^{\prime}$ end using cutadapt version 1.13 [14]. Remaining reads were further filtered by BWA-MEM version 0.7.13-r1126, paired-end mode [15] and samtools version 1.3.1 with htslib 1.3.1 [16] to remove reads mapped to human genome (GRCh38) and Phix genome. Read pairs with a read mean quality score below 30 or a read length shorter than $75 \%$ of the read length (i.e., 105 bp) were also discarded. FastQCv0.11.7 [17] was performed on the remaining reads (Pass Filter Reads). Paired-end FASTQ files were separately merged in case of multiple read files for the same sample. Analysis of the taxonomic distribution was performed by MetaPhIAn2 v2.0 [18], a profiling algorithm using clade-specific marker genes. All other analyses were performed in RStudio using the package ggpubR [19].

\section{Results \& discussion}

Shotgun or untargeted metagenomics is a powerful tool that is being used to interrogate complex microbial communities and obtain actionable insights [7]. Application of this tool towards developing products and services necessitates performing quality control on the workflow. Here, we provide one tool that helps address this need spiking-in DNA from known organisms in a defined ratio at the very beginning of the metagenomics workflow (cell lysis) and then calculating the ratio at the end (bioinformatics analysis). A correspondence between the input and output ratio would be one indicator of the quality of our workflow. The spike-in is a mixture of DNA from $A$. fischeri and $R$. palustris in a ratio of 4:1. These organisms were chosen: i) to capture potential GC bias in the workflow; and, ii) because their genomes are sequenced and well characterized thereby facilitating bioinformatic analyses. It is also important to point out that these organisms have been developed and used as spike-in

(A)

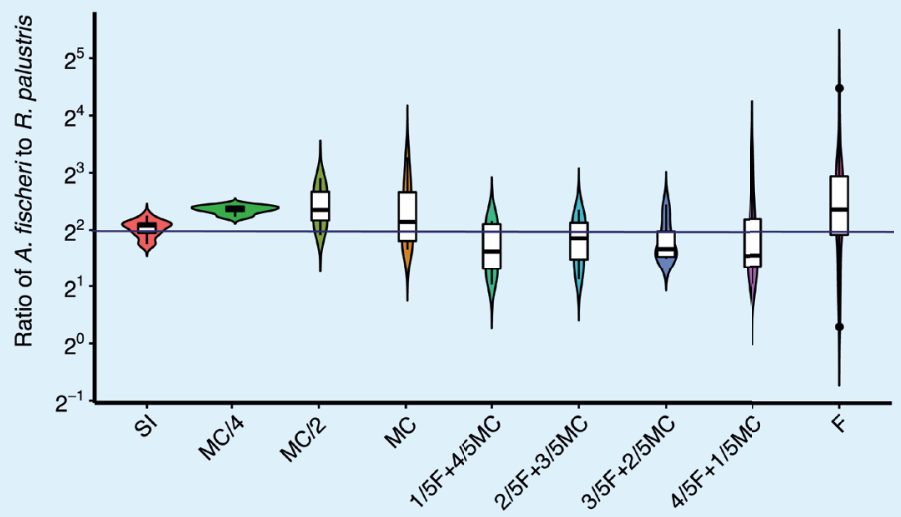

(B)

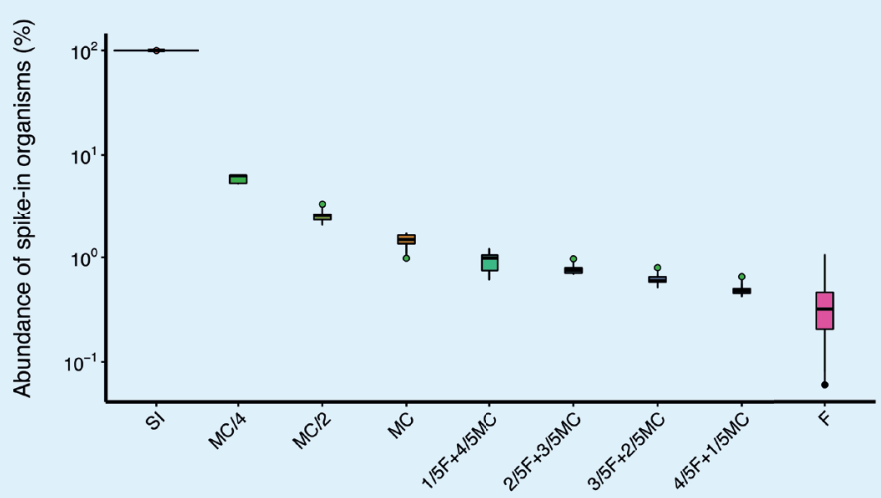

(C)

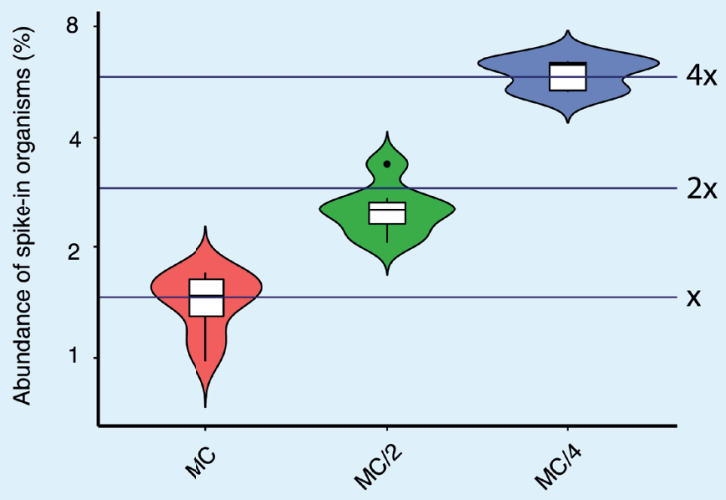

Figure 1. Spike-in genomic DNA can be used to validate metagenomics workflows. (A) Overall, the output ratio of the spike-in matches with the input ratio in samples of varying diversities. The solid line is the expected ratio - 4. (B and C) Spike-in reads are inversely and linearly related to the microbial load of the samples. Data shown in (C) is a subset of the data in (B). The expectation regarding the linear relationship of spike-in abundances with microbial load is better tested in dilutions of the defined mock community. The horizontal intercepts denote the median relative abundances. (A and C) Colored areas represent violin plots and whiskerplots represent boxplots. (B) Colored areas represent whiskerplots. The figures are all generated using the $\mathrm{R}$ package ggplot2 as part of ggpubR.

F: Fecal material; MC: Undiluted mock community; MC/4: Mock community diluted fourfold; $\mathrm{MC} / 2$ : Mock community diluted twofold; SI: Spike-in added to sterile saline. 
for 16S-rRNA encoding gene surveys by Stammler et al. [13]. We chose DNA over whole cells because of the ease of quantitation, implementation and reproducibility versus whole cells.

In order to determine if the spike-in is a useable approach to validate metagenomics workflow(s), some basic assumptions are that the spike-in should be reliably quantified, easily distinguishable from the microbial diversity of the actual sample, and not swamp out the signal from the sample. We tested this assumption by adding the spike-in DNA mix to multiple replicates from multiple samples of varying complexity: sterile saline, defined mock community and fecal material, and processing them through the entire workflow starting from DNA extraction. In all cases, sequencing reads were assigned to the spike-in DNA (A. fischeri and $R$. palustris) and could be easily separated from the microbial diversity of the actual samples over a wide range of relative abundances (Figure S1). In the case of spike-in added to just sterile saline, 100\% of the sequencing reads were assigned to just the spike-in organisms (Figure S1A). This clearly demonstrates that no crosscontamination occurred during sample processing and library preparation and that there was no background contamination from the reagents. Importantly, the spike-in organisms were not detected in unspiked samples.

After confirming that the spike-in DNA was identified via sequencing, our next goal was to determine how well the output ratio of the spike-in met the input ratio. The spike-in consisted of DNA from $A$. fischeri and $R$. palustris mixed in a ratio of $4: 1$ and added to samples. If the workflow is robust, we expect the output ratio of the relative abundances of these organisms (corrected for genome sizes) to be close to 4 . This expectation was tested across samples of varying complexities ranging from sterile saline all the way to fecal material. Spike-in mixed with sterile saline is the control case (SI). Indeed, in this case, the mean output ratio of relative abundances of the two spike-in organisms was $4.08(95 \% \mathrm{Cl}$ [3.81,4.34]; $n=12$; Figure 1A). To increase the sample complexity, the spike-in mix was added to a synthetic mock community of bacteria and fungi and processed through the entire workflow (MC). Multiple dilutions of the mock community were also prepared and spike-in was added to them as well (1:2 dilution - MC/2; 1:4 dilution - MC/4).
This enabled us to test the metagenomics workflow with samples of different but defined biomass. Again, in the case of the mock community samples, the mean output ratio was close to $4(5.26 ; 95 \% \mathrm{Cl}$ [4.56,5.95]; $n=24$; Figure 1A). Next, to increase the complexity further, the spike-in DNA mix was added to varying mixes of mock community and fecal material (from 4/5th mock + 1/5th fecal all the way to $1 / 5$ th mock + 4/5th fecal). In these backgrounds, the output ratio was 3.58 (95\% Cl [3.16,4.00]; $\mathrm{n}=10$ for each mix of mock community and fecal material; Figure 1A). Finally, the DNA spike-in mix was added directly to the fecal material. Despite the complexity and heterogeneity of these samples, the output ratio of the spike-in DNA was $5.99(n=33 ; 95 \% \mathrm{Cl}$ [4.56,7.42]; Figure 1A). It is important to point out that the deviation from the expected ratio of 4:1 was higher in the case of these samples; again, this is to be expected since fecal material is very heterogenous and contains potential reaction inhibitors such as bile acids and other small molecules. It is possible that these components play a role in exacerbating GC-bias. Overall, on average, regardless of the sample type (total $n=110$ ), the mean spike-in output ratio was
4.73 (95\% Cl [4.23,5.24]). The performance of the spike-in did not have a relationship with sample diversity (number of species detected).

Thus far, we used the spike-in mix ratio as a QC metric of our workflow and demonstrated good correspondence between the input and output ratios. Another expectation with a robust workflow is that the relative abundance of the spike-in organisms should be inversely related to the microbial load of the sample; that is, more reads from the spike-in organisms with less microbes in the background. If this expectation is true, then the abundance of the spike-in organisms should be highest with the spike-in by itself (SI) and then gradually decrease along the spectrum of increasing microbial load all the way to fecal material. Indeed, this expectation is fulfilled (Figure 1B). The expectation can be refined further when working with samples with defined microbial loads. We took advantage of the mock community samples and dilutions thereof to test this expectation. The mock community diluted 1:2 (MC/2) should contain half as many microbes as the undiluted mock community (MC) and the MC/4 samples should contain a quarter of the microbes.

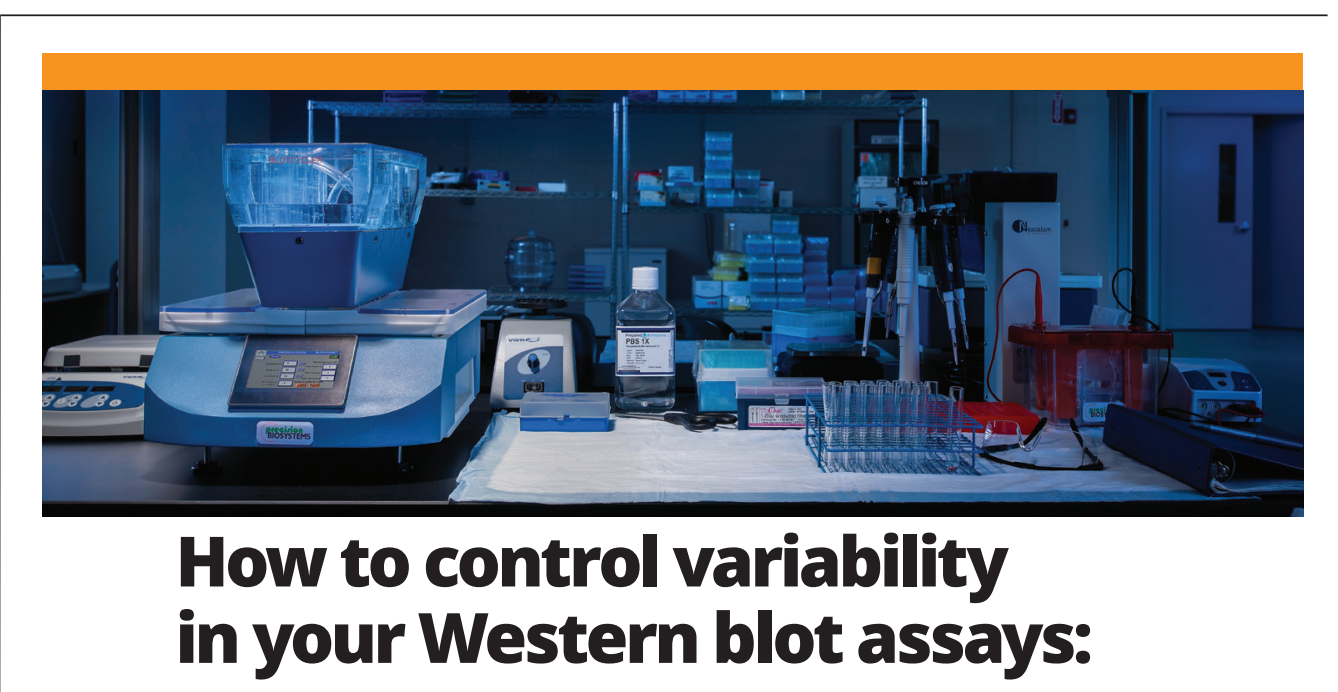

\author{
By eliminating manual errors in fluid delivery, timing
} and washing, BlotCycler ${ }^{\text {TM }}$ consistently delivers reproducible Western blot results. BlotCycler performs every step in the

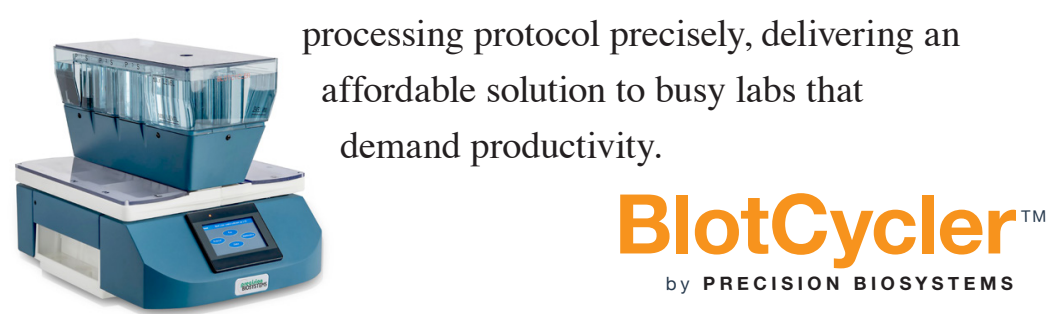

www.blotcycler.com 


\begin{tabular}{l} 
Stage 1 \\
1. Break workflow into discrete modules, e.g., DNA \\
extraction and library preparation. \\
2. Add spike-in genomic DNA to the sample of \\
interest at the first step of the module. For instance, spike-in \\
before tagmentation if library preparation is the module. \\
3. Module with the maximum deviation from \\
expected ratio is identified and iterated upon for \\
improvement. \\
4. Put modules together and use spike-in to validate \\
the entire workflow (Stage 2). \\
Stage 2 \\
1. Assess variance in the spike-in ratio using the experimental \\
design outlined (Figure 1). \\
2. Spike-in ratio should be closest to the expected value \\
when the spike-in genomic DNA is added to sterile saline \\
and processed through the workflow. \\
3. Test the spike-in performance in samples of varying \\
complexities. Ensure that these samples include \\
microbiomes of interest. Defining the acceptable \\
variance is left to the operator's discretion. Based \\
upon all the samples described here, we defined \\
the acceptable range to be between 4 and 5.4 \\
(99\% confidence intervals). \\
Solage 3 \\
1. Include triplicates of just the spike-in added to sterile \\
saline as positive control. \\
of interest. The spike-in genomic DNA can be added \\
to this pool in triplicate and processed through the \\
workflow. Spike-in performance is calculated as \\
outlined in Stage 2. \\
\hline
\end{tabular}

Figure 2. Suggested staged approach towards using the spike-in genomic DNA for monitoring and validating metagenomics workflows.

QC: Quality control.

Therefore, if the relative abundance of the spike-in organisms in the undiluted mock community is $x$, then the relative abundance should be doubled in the mock community diluted 1:2 (2x) and quadrupled in the mock community diluted 1:4 (4x). This expectation was met as well (Figure 1C).

In summary, the successful development of spike-in genomic DNA and the quality of our metagenomics workflow is demonstrated by three simple measures. First, the spike-in is clearly identifiable in the background of different kinds of samples (Figure S1A). Second, when the spike-in is added to samples of varying complexity, ratio of the relative abundances is close to what is expected (Figure 1A). The relative abundances of the spike-in themselves are inversely related to the microbial load of the samples (Figure 1B \& C). As such, we did not observe a pronounced GC-bias in the workflow since this would have led to an under-representation of $R$. palustris and resulted in the ratio being greater than 4:1. Another attractive feature of this spike-in approach is that typically shotgun metagenomic survey analyses involves interrogating relative abundances and therefore a QC metric that is a relative abundance measure in itself is valuable.

The use of a spike-in approach for QC in sequencing RNA and DNA has a long history and its importance has been echoed in multiple publications [20,21]. Many recent publications have highlighted the use of the spike-in for 16S-rRNA encoding gene surveys, both for $\mathrm{QC}$ as well as a normalization tool to move from relative towards absolute abundance $[13,22,23]$. We built upon these studies to demonstrate the use of a spike-in for validating a shotgun/ untargeted metagenomics workflow. To the best of our knowledge, this is the first application of a spike-in approach for validating shotgun metagenomics workflows. A staged approach for employing the spike-in genomic DNA to monitor and validate metagenomics workflows is presented in Figure 2. The spike-in approach could have three possible uses: i) validating the entire workflow; ii) QC on a per-sample basis; and, iii) normalization of reads by microbial load. QC on a per-sample basis with the spike-in and normalization to microbial loads both require fulfilling an assumption that the spike-in will not interfere with the microbial composition of the actual sample. This assumption is hard if not impossible to meet. Even if this assumption is met at a taxonomy level, the spike-in DNA could confound inferring the functional composition (gene and pathway abundance) from shotgun metagenomic surveys, especially for genes and pathways that are widespread among bacteria such as glycolysis. Hence, we believe and recommend that the most tractable use of the spike-in is to validate the metagenomics workflow, either end-toend from cell lysis to bioinformatics analyses or from library preparation to bioinformatic analyses (Figure 2). This approach is relevant, simple, inexpensive and easily implemented in a laboratory setting. We anticipate that this will be a community resource that can be used widely in laboratories for validating their respective workflows.

\section{Author contributions}

AV, MP, PH, DS, XW and JPT designed the experiments. AV and MP performed the experiments. AV, $\mathrm{PH}, \mathrm{XW}$ and $\mathrm{DS}$ performed the analyses. All authors reviewed and approved the manuscript. 


\section{Financial \& competing interests disclosure}

This work was supported in its entirety by Procter \& Gamble. The authors are employees of Procter \& Gamble. The authors have no other relevant affiliations or financial involvement with any organization or entity with a financial interest in or financial conflict with the subject matter or materials discussed in the manuscript apart from those disclosed.

No writing assistance was utilized in the production of this manuscript.

\section{Open access}

This article is distributed under the terms of the Creative Commons AttributionNonCommercial-NoDerivatives 4.0 Unported License. To view a copy of the license, visit http://creativecommons.org/ licenses/by-nc-nd/4.0/

\section{Supplementary data}

To view the supplementary data that accompany this paper please visit the journal website at: www.future-science. com/doi/suppl/10.2144/btn-2018-0089

\section{References}

1. Cheng PM. Power unseen: how microbes rule the world. J. Biol. Med. 67(1-2), 54-55 (1994).

2. O'Toole PW, Paoli M. The contribution of microbial biotechnology to sustainable development goals: microbiome therapies. Microb. Biotechnol. 10(5), 1066-1069 (2017).

3. Tshikantwa TS, Ullah MW, He F, Yang G. Current trends and potential applications of microbial interactions for human welfare. Front. Microbiol. 9(1156), (2018).

4. Umesha S, Singh PK, Singh RP. Chapter 6 - Microbial biotechnology and sustainable agriculture. In: Biotechnology for Sustainable Agriculture. Singh RL, Mondal S (Eds). Woodhead Publishing, 185-205 (2018).

5. Timmis K, De Vos WM, Ramos JL et al. The contribution of microbial biotechnology to sustainable development goals. Microb. Biotechnol. 10(5), 984-987 (2017).

6. Bouchie A. White House unveils National Microbiome Initiative. Nat. Biotechnol. 34, 580 (2016).

7. National Research Council Committee on Metagenomics C, Functional A. The National Academies Collection: Reports funded by National Institutes of Health. The New Science of Metagenomics: Revealing the Secrets of Our Microbial Planet doi:10.17226/11902 (2007).

8. Debelius J, Song SJ, Vazquez-Baeza Y, Xu ZZ, Gonzalez A, Knight R. Tiny microbes, enormous impacts: what matters in gut microbiome studies? Genome Biol. 17(1), 217 (2016).

9. Costea PI, Zeller G, Sunagawa S et al. Towards standards for human fecal sample processing in metagenomic studies. Nat. Biotechnol. 35(11), 1069-1076 (2017).

10. Mcintyre ABR, Ounit R, Afshinnekoo E et al. Comprehensive benchmarking and ensemble approaches for metagenomic classifiers. Genome Biol. 18(1), 182 (2017).

11. Mason CE, Afshinnekoo E, Tighe S, Wu S, Levy S. International Standards for Genomes, Transcriptomes, and Metagenomes. J. Biomol. Tech. 28(1), 8-18 (2017).

12. Vandeputte D, Kathagen G, D'hoe K et al. Quantitative microbiome profiling links gut community variation to microbial load. Nature 551(7681), 507-511 (2017).

13. Stammler F, Glasner J, Hiergeist $A$ et al. Adjusting microbiome profiles for differences in microbial load by spike-in bacteria. Microbiome 4(1), 28 (2016).

14. Chen $\mathrm{C}$, Khaleel SS, Huang $\mathrm{H}$, Wu CH. Software for pre-processing Illumina next-generation sequencing short read sequences. Source Code Biol. Med. 9, 8 (2014).

15. Li H, Durbin R. Fast and accurate short read alignment with Burrows-Wheeler transform. Bioinformatics 25(14), 1754-1760 (2009).

16. Li H, Handsaker B, Wysoker A et al. The sequence alignment/map format and SAMtools. Bioinformatics 25(16), 2078-2079 (2009).

17. Brown J, Pirrung M, Mccue LA. FQC Dashboard: integrates FastQC results into a web-based, interactive, and extensible FASTQ quality control tool. Bioinformatics doi:10.1093/bioinformatics/ btx373 (2017).

18. Truong DT, Franzosa EA, Tickle TL et al. MetaPhIAn2 for enhanced metagenomic taxonomic profiling. Nat. Methods 12(10), 902903 (2015).

19. Kassambara A. ggpubR: 'ggplot2' based publication ready plots. (2018).

20. Chen K, Hu Z, Xia Z, Zhao D, Li W, Tyler JK. The overlooked fact: fundamental need for spike-in control for virtually all genome-wide analyses. Mol. Cell. Biol. 36(5), 662-667 (2016).

21. Satinsky BM, Gifford SM, Crump BC, Moran MA. Use of internal standards for quantitative metatranscriptome and metagenome analysis. Methods Enzymol. 531, 237-250 (2013).

22. Tourlousse DM, Yoshiike S, Ohashi A, Matsukura S, Noda N, Sekiguchi Y. Synthetic spike-in standards for high-throughput 16S rRNA gene amplicon sequencing. Nucleic Acids Res. 45(4), e23-e23 (2017).

23. Smets W, Leff JW, Bradford MA, Mcculley RL, Lebeer S, Fierer N. A method for simultaneous measurement of soil bacterial abundances and community composition via 16S rRNA gene sequencing. PeerJ PrePrints 3, e1318v1311 (2015).

First draft submitted: 2 July 2018; Accepted for publication: 29 August 2018

Address correspondence to: Arvind Venkataraman; The Procter \& Gamble Company, 8700 Mason Montgomery Road, Mason, $\mathrm{OH}$ 45040, USA; venkataraman.a@pg.com

To purchase reprints of this article contact: s.cavana@future-science.com
SPEX SamplePrep?

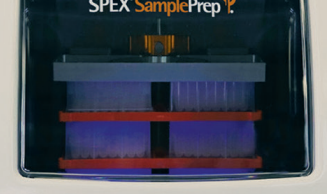

() OFENO/GRINDER

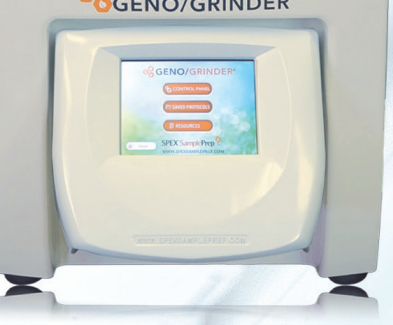

The Geno/Grinder ${ }^{\circledR}$ is a high-throughput plant and animal tissue homogenizer specifically designed for rapid cell disruption, lysis and tissue homogenization.

- Adjustable clamp that accommodates up to six deep-well titer plates, or multiple sample tubes from $2 \mathrm{~mL}$ to $50 \mathrm{~mL}$.

- Programmable touch screen control panel that saves up to 500 protocols.

SPEX SamplePrep P

(+1) 732-623-0465

learnmore@spex.com

SPEXSAMPLEPREP.COM/GENOGRINDER 
